\title{
DETERMINAÇÃO DA CONDUTIVIDADE E DIFUSIVIDADE HIDRÁULICA DOS SOLOS TERRA ROXA ESTRUTURADA E LATOSSOLO ROXO ATRAVÉS DA INFILTRAÇÃO VERTICAL ${ }^{a}$
}

\author{
CR. APPOLONI \\ J.C.M. DE OLIVEIRA ${ }^{\mathrm{C}}$ \\ L. FANTE JR. ${ }^{d}$ \\ A.D.B. DESOUZA \\ J.M. DE OLIVEIRA JR. ${ }^{\dagger}$
}

\begin{abstract}
RESUMO
Foram calculadas as funções condutividade e difusividade hidráulica em amostras de solo (horizontes A e B), de um latossolo roxo (LRJ e uma terra roxa estruturada (TE) da região de Londrina-PR, a partir do perfil de umidade e de dados da propagação da frente de molhamento, obtidos através da medida da infiltração de água em coluna de solo e de um equacionamento variacional do escoamento vertical. Nas medidas da frente de molhamento utilizou-se um tubo de acrílico, contendo a amostra de solo, acoplado a uma placa porosa em sua base, permitindo a infiltração da água em sentido contrário ao campo gravitacional, com uma velocidade de $0,12 \mathrm{~cm} / \mathrm{min}$. Os perfis de umidade foram obtidos pela técnica de atenuação de raios gama provenientes de uma fonte de $\mathrm{Co}^{60}$, medidos por um detector de cintilação NaI (TL). O conjunto fonte-detector foi montado numa mesa que permitia a movimentação vertical e horizontal da coluna.
\end{abstract}

PALAVRAS-CHAVE: infiltração vertical, atenuação de raios gama, condutividade e difusividade hidráulica.

\section{1 - INTRODUÇÃO}

O uso, aplicação e manejo de água no solo, tem sido estudados através de diferentes pesquisas hidrológicas realizadas em todo mundo, e envolvem o conhecimento de uma série de propriedades físico-hídricas do solo.

Ao estudar o ciclo da água na agricultura nos deparamos com o processo de infiltração, cuja compreensão é fundamental para o planejamento de projetos de irrigação e para estabelecer medidas para o controle de erosão através da diminuição do deflúvio superficial.

A medida da condutividade hidráulica é essencial para a caracterização físico-hídrica dò solo e a quantificação do movimento da água, permitindo o cálculo dos fluxos. Como existem uma alta dependência entre o conteúdo de água no solo e as funções condutividade hidráulica $[\mathrm{K}(q)$ e difusividade [ $\mathrm{D}(q)$ da água, estas tornam de difícil determinação.

Desta maneira o objetivo do presente trabalho foi determinar o perfil de umidade em função do tempo, numa coluna de solo e posteriormente, a partir destes dados obter as funções $\mathrm{K}(q)$ e $\mathrm{D}(q)$ da amostra de solo estudado.

\section{2 - MATERIAL E MÉTODOS}

Arranjo Experimental - Neste experimento um item de fundamental importância é a criação de um dispositivo de fornecimento de água à coluna de solo, de maneira a produzir-se uma infiltração com velocidade e uniformidade adequadas.

O arranjo experimental constitui-se de uma fonte de $\mathrm{Co}^{60}$, detector de Nal (TL), mono-canal, pré-amplificador, contador, cronômetro e uma mesa (Figura 1). A linha gama usada foi a de $1,17 \mathrm{MeV}$ do $\mathrm{Co}^{60}$, para trabalharmos com feixe monoenergético.

A interface água-amostra de solo consistiu de uma placa de acrílico $(15,0 \mathrm{~cm}$ de diâmetro) com orifícios de $1 \mathrm{~mm}$ de diâmetro. Junto com a placa de acrílico foram colocadas camadas de tecido (Nylon) e uma fita de zinco com borracha, visando conferir coesão com a base do cilindro de acrílico $(14,5 \mathrm{~cm}$ de diâmetro x $30 \mathrm{~cm}$ de altura). A infiltração revelou-se uniforme, com uma velocidade adequada $(0,12 \mathrm{~cm} / \mathrm{min})$.

Este conjunto foi acoplado a um suporte que mantinha a placa porosa em contado com a água.

Após uma prévia secagem ao ar o solo foi peneirado (peneira com malha $2 \mathrm{~mm}$ ) e colocado no tubo, sem compactação.

A manipulação da amostra de solo foi feita com o auxílio de uma mesa com um dispositivo que permite movimentação horizontal e vertical. Dessa maneira foi possível irradiar o solo em incrementos de $2 \mathrm{~cm}$, até $20 \mathrm{~cm}$ da coluna de solo.

a - Trabalho realizado no Departamento de Física/CCE - Universidade Estadual de Londrina, Londrina-PR.

b - Professor Adjunto do Departamento de Física da Universidade Estadual de Londrina, Caixa Postai 6001, CEP 86051, Londrina-PR.

c - Bolsista do CNPq, categoria aperfeiçoamento.

d - Físico, pós-graduando no Departamento de Física Nuclear, Instituto de Física, USP (SP); bolsista do CNPq.

e - Aluno de Graduação do Curso de Física da Universidade Estadual de Londrina.

f - Físico, pós-graduando no Departamento de Física Nuclear, Instituto de Física, USP (SP); bolsista do CNPq. 


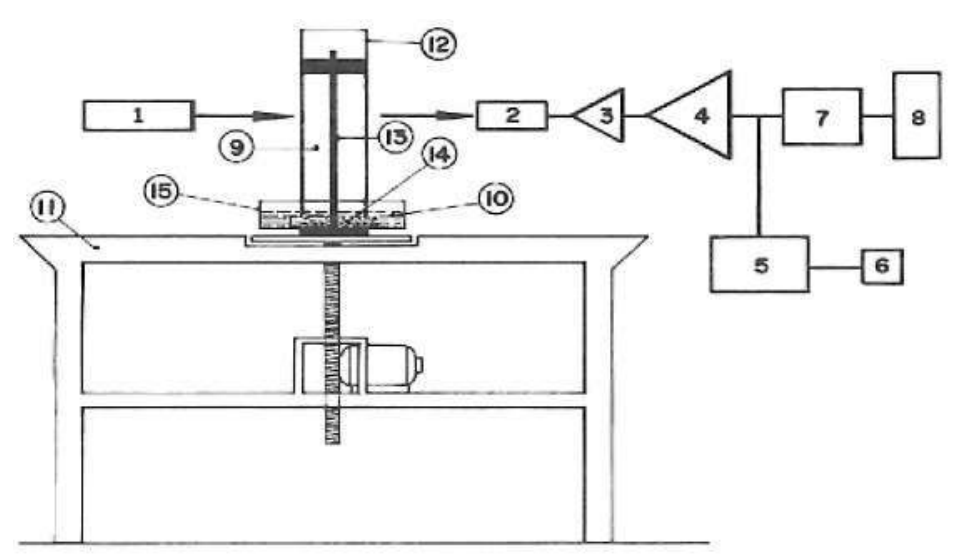

FIGURA 1 - Sistema de infiltração vertical de água no solo, acoplado à aparelhagem padrão.

1. Fonte de ${ }^{60} \mathrm{Co}$

2. Detector de cintilação Nal (TL)

3. Pré-amplificador

4. Amplificador

5. Analisador multi-canal

6. Osciloscópio

7. Analisador mono-canal

8. Contador

9. Solo

10. Água

11. Mesa de medidas

12. Cilindro de acrílico

13. Suporte

14. Placa porosa

15. Reservatório

Obtenção dos Dados - Na fase de obtenção dos dados, mediu-se a intensidade ( $\left.\mathrm{I}_{0}\right)$ sem absorvedor. Em seguir da varreu-se o tubo de acrílico sem a amostra de solo, determinando-se assim, a intensidade do feixe transmitido através do tubo vazio $\left(I_{v}\right)$ para cada posição, a qual foi variada em incrementos de $2 \mathrm{~cm}$. A intensidade $I_{0}$ foi novamente medida e em seguida colocou-se a amostra de solo (preparada conforme o item interior) no tubo, medindo-se então a intensidade $\left(I_{\mathrm{s}}\right)$ para cada posição.

As intensidades para cada posição foram medidas durante aproximadamente $3 \mathrm{~min}$. e ao final de cada seqüência de 10 posições era novamente obtida a intensidade sem absorvedor. Este procedimento foi feito até que a última posição estivesse saturada.

A umidade do solo ( qo ) foi previamente determinada pelo método gravimétrico.

A densidade global da amostra foi calculada pela razão entre a massa e o volume da amostra.

Análise dos Dados - Através da varredura da amostra com o feixe de raios gama, calculou-se o coeficiente de atenuação de massa do solo ( $\mathrm{msrs}$ ) dado por:

$\mu_{\mathrm{s}} \rho_{\mathrm{s}}=\frac{1}{\mathrm{x}} \ln \left[\frac{\mathrm{I}_{\mathrm{o}^{1}} \cdot \mathrm{I}_{\mathrm{V}}}{\mathrm{I}_{\mathrm{o}} \cdot \mathrm{I}_{\mathrm{S}}}\right]-\mu_{\omega} \rho_{\omega} \cdot \theta_{\mathrm{o}}$ onde: $\mathbf{x}$ - expessura da amostra

$I_{0^{1}}$ - intensidade sem absorvedor no final da mesma sequência

$\mu_{\omega} \rho_{\omega}$ - coeficiente de atenuação de massa da água (obtido da literatura - Fetraz, 1974)

A partir destes dados calculou-se $\theta(\mathrm{z}, \mathrm{t})$ utilizando-se a expressato abaixo:

$\theta(z, t)=\left[\frac{1}{x} \ln \left[\frac{I_{o^{11}} \cdot I_{v}}{I_{0^{1}} \cdot I}\right]-i s_{s} \rho_{\mathrm{s}}\right] \frac{1}{\mu_{\omega} \rho_{\omega}}$

onde: $\mathrm{I}_{0^{11}}$ - intensidade do feixe que emerge da amostra para o detector a as demais variaveis são as mesmas detalhadas no indice da equaçăo (1).

0 desvio nos valores experimentais de $\mu_{\mathrm{S}} \rho_{\mathrm{S}}$ e $\theta(\mathrm{z}, \mathrm{t})$ foi calculado através da propagaçăo de erros nas equaçōes (1) e (2). Através do gráfico de $\theta(z, t)$ versus tempo, para cada posiçăo, determùiou-se a umidade de saturaçao $\left(\theta_{\mathrm{s}}\right)$ e o tempo de saturaçao ( $\left.t_{s}\right)$ para cada posiçao $\left(X_{S}\right)$.

Para a determinaçăo dos parametyos de difusividade e condutividade hidráulicas utilizou-se uma soluçao da equação de Darcy para meios homogêneos (Reichardt, 1985).

Através da relaçao entre $X_{s} / \sqrt{t_{s}} \in \sqrt{t_{s}}$ foi ohtida a seguinte equação:

$$
\frac{x_{s}}{\sqrt{t_{s}}}=b_{1} \sqrt{t_{s}}+a_{1}
$$

A partir de um determinado tempo de referencia, foram obtidos do gráfico $\theta(\mathrm{z}, \mathrm{t})$ versus o tempo os seguintes. elementos: a umidade inicial $\left(\theta_{o \gamma}\right)$, a umidade $\theta$, o parânetro $\eta=\mathrm{z} / \mathrm{q}$ (onde: $\mathrm{z}$ é a posiçăo $(\mathrm{cm})$ de cada nível e q é a posiçāo $(\mathrm{cm})$ da frente de molhamento $)$ e $\omega=\left(\theta-\theta_{o \gamma}\right) /$ ( $\left.\theta \mathrm{s}-\theta_{\mathrm{o} \gamma}\right)$ é um parâmetro adimensional (derivado da umidade) para cada posiçăo no referido tempo.

A partír do gráfico da função $\ln (1-\eta) \times \omega$ estabelecelı-se a equaçāo:

$$
\ln (1-\eta)=b_{2} \omega+a_{2}
$$

Desta forma, Coutinho Jr. et al., 1977, utilizando-se das equaçōes (3) e (4) e as equaçסes abaixo:

$$
\begin{aligned}
& F(\beta)=\mathrm{e}^{\beta}(\beta-7 / 3)-2 \mathrm{e}^{\beta / 2} \text { arctge } \beta / 2 \\
& \gamma(\beta)=\beta^{2}-6^{\beta}+34 / 3 \\
& C(\beta)=\left(\frac{6 \beta-13)}{18} \mathrm{e}^{\beta}+\frac{\pi}{3} \mathrm{e}^{\beta / 2}\right.
\end{aligned}
$$

onde:

$$
\beta=a_{2}+b_{2}
$$

calcularam respectivamente a difusividade e condutividade hidráulica do solo pelas equaçoes:

$$
D(\omega)=D_{o} e^{\beta \omega}
$$




$$
K(\omega)=K_{0} e^{\beta \omega}
$$

onde:

$$
\begin{aligned}
& D_{0}=\frac{a_{1}{ }^{2} \gamma(\beta)}{2 F(\beta)} \\
& K_{o}=\frac{3 b_{1} \gamma(\beta)}{2 C(\beta)}
\end{aligned}
$$

Para transformar $\mathrm{D}(\omega)$ em $\mathrm{D}(\theta)$ é necessário fazer a seguinte transformaçđo:

$$
\begin{aligned}
& \mathrm{D}(\omega)=\mathrm{D}_{\mathrm{o}} \text { e } \beta \omega \\
& \mathrm{D}(\omega)=\mathrm{D}_{\mathrm{o}} \quad \operatorname{ExP}\left[\beta\left[\begin{array}{l}
\theta-\theta_{\mathrm{o} \gamma} \\
\theta_{\mathrm{s}}-\theta_{\mathrm{o} \gamma}
\end{array}\right]\right.
\end{aligned}
$$

chamando

$$
\begin{aligned}
& \gamma=\frac{\beta}{\theta_{\mathrm{s}}-\theta_{\mathrm{o} \gamma}} \\
& \mathrm{D}(\theta)=\mathrm{D}_{\mathrm{o}} \mathrm{e} \gamma\left(\theta-\theta_{\mathrm{o} \gamma}\right) \\
& \mathrm{D}(\theta)=\mathrm{D}_{\mathrm{o}} \mathrm{e} \cdot \mathrm{e} \gamma\left(\theta-\theta_{\mathrm{s}}\right) \\
& \mathrm{D}(\theta)=\mathrm{D}_{\mathrm{o}}^{1} \text { e } \gamma\left(\theta-\theta_{\mathrm{s}}\right) \mathrm{cm}^{2} / \mathrm{min} \\
& \quad \operatorname{com} \mathrm{D}_{\mathrm{o}}^{1}=\mathrm{D}_{\mathrm{o}} \mathrm{e} \beta
\end{aligned}
$$

Analogicarnen te para $\mathrm{K}(\omega)$ teremos a expressao

$$
\mathbf{K}(\theta)=\mathbf{K}_{\mathrm{O}}^{\mathrm{I}} \text { e } \gamma\left(\theta-\theta_{\mathrm{s}}\right) \mathrm{cm} / \mathrm{min}
$$

com

$$
\mathbf{K}_{\mathbf{0}}^{1}=K_{0} e^{\beta}
$$

Os cálculos foram realizados multiplicando as equaços acima por 1440 para obtermos $D(\theta)$ e $K(\theta)$ em $\mathrm{cm}^{2} / \mathrm{dia}$ e $\mathrm{cm} / \mathrm{dia}$ respectivamente.

\section{3 - RESULTADOS E DISCUSSÃO}

No quadro 1 são apresentados os valores médios de " $\rho_{\mathrm{s}} \rho_{\mathrm{s}}$ para cada horizonte dos solos estudadoi. A densidade destes variou de 1.03 a $1.05 \mathrm{~g} / \mathrm{cm}^{3}$. Com esses dados foi possivel traçar as curvas de molhamen to $\theta(2, t)$ versus $t$, , $A$ partir da anälise das curvas de molhamento (Figura 2), ajustou-se o modelo citado no item Análise de Dados e foram construidos os gráticos $X_{s} / \sqrt{t_{s}}$ versus $\sqrt{t_{s}}$ en $\left.\ln -\eta\right)$ versus $\omega$ para cada amostra de solo. como mostram as figuras 3 e 4, respectivamente.

Com base nas parametrizaçoes destas retas foi possivel o cálculo da difusividade $[D(\omega)$ j c condutividadk $[K(\omega)$ ], cujos valores médios sáo mostrad́dos no quadro 2 , sendo que as respectivas curvas de $D(\theta)$ e $K(\theta)$ são apresentadas nas figuras $S$ e 6 .

Lembrando-se que $\mathrm{D}(\omega)$ e $\mathrm{K}(\omega)$ sio funçōes exponenciais de 3 . pode-se observar que para o solo LR-A. o parâmetro $\beta(7,22)$ é 30 a $40 \%$ maior em relação ao LR-B $(\beta=5,53)$, bem como aos horizontes $\mathrm{A}$ e $\mathrm{B}$ do solo TR (respectivamente $\beta=4.96$ e 4,37). Decorrendo assitn que $o$

\begin{tabular}{|c|c|c|c|}
\hline Solo & Horizonte & Condutivid & Difusividade D $(\omega)$ \\
\hline
\end{tabular}
horizonte A do latossolo Roxo tem $\mathrm{D}(\theta)$ e $\mathrm{K}(\theta)$ sensivelmente maiores que o horizonte $\mathrm{B}$ e também maior do que para o solo TE.

Para o solo TE observou-se pouca diferença no valor de $\beta$ entre os horizontes $A(\beta=4.96)$ e $B(\beta=4.37)$, as-

\section{QUADRO 1 - VALORES MÉDIOS DO COEFICIENTE DE MASSA DOS SOLOS $\left(\mu_{\mathrm{S}} \rho_{\mathrm{S}}\right)$ E SEUS RES- PECTIVOS DESVIOS $\left(\sigma \mu_{\mathrm{S}} \rho_{\mathrm{S}}\right)$}

\begin{tabular}{lccc}
\hline Solo & Horizonte & $\mu_{\mathrm{s}} \rho_{\mathrm{s}}$ & $\alpha \mu_{\mathrm{s}} \rho_{\mathrm{s}}$ \\
\hline & & \multicolumn{2}{c}{$\mathrm{cm}^{-1}$} \\
LR & $\mathrm{A}$ & 0.0502 & 0.0006 \\
LR & $\mathrm{B}$ & 0.0455 & 0.0015 \\
TE & $\mathrm{A}$ & 0.0497 & 0.0014 \\
TE & $\mathrm{B}$ & 0.0495 & 0.0020 \\
\hline
\end{tabular}

QUADRO 2 - VALORES MÉdIOS PARA CONDUTIVIDADE [ K $(\omega)$ ] E DIFUSIVIDADE [ D ( $\omega)$ ] HIDRÁULICA PARA OS SOLOS ESTUDADOS.

$\mathrm{cm} /$ minuto

$\begin{array}{lll}\text { LR } & \text { A } & K(\omega)=1,49 \cdot 10^{-3} \operatorname{EXP} 7,22 \omega \\ \text { LR } & \text { B } & K(\omega)=1,60 \cdot 10^{-2} \operatorname{EXP} 5,53 \omega \\ \text { TE } & \text { A } & K(\omega)=2,90 \cdot 10^{-3} \operatorname{EXP} 4,96 \omega \\ \text { TE } & \text { B } & K(\omega)=7,00 \cdot 10^{-3} \operatorname{EXP} 4,37 \omega\end{array}$

$\mathrm{cm}^{2} /$ minuto

$D(\omega)=2,27 \cdot 10^{-2} \operatorname{EXP} 7,22 \omega$

$\mathrm{D}(\omega)=3,75 \cdot 10^{-2} \operatorname{EXP} 5,53 \omega$

$\mathrm{D}(\omega)=5,14 \cdot 10^{-3} \operatorname{EXP} 4,96 \omega$

$D(\omega)=1,33 \cdot 10^{-2} \cdot \operatorname{EXP} 4,37 \omega$ 
sim sendo, a difusividade e condutividade hidráulica dos dois horizontes são semelhantes.

Deve-se lembrar que, para este trabalho, os solos foram peneirados e desta forma alterou-se a matriz original do solo, sendo que os horizontes, A e B, ficaram relativamente mais parecidos. Para se fazer uma melhor análise dever-se-ia conhecer alguns parâmetros dos solos, como granulometria e teor de matéria orgânica, o que neste caso não foi realizado.

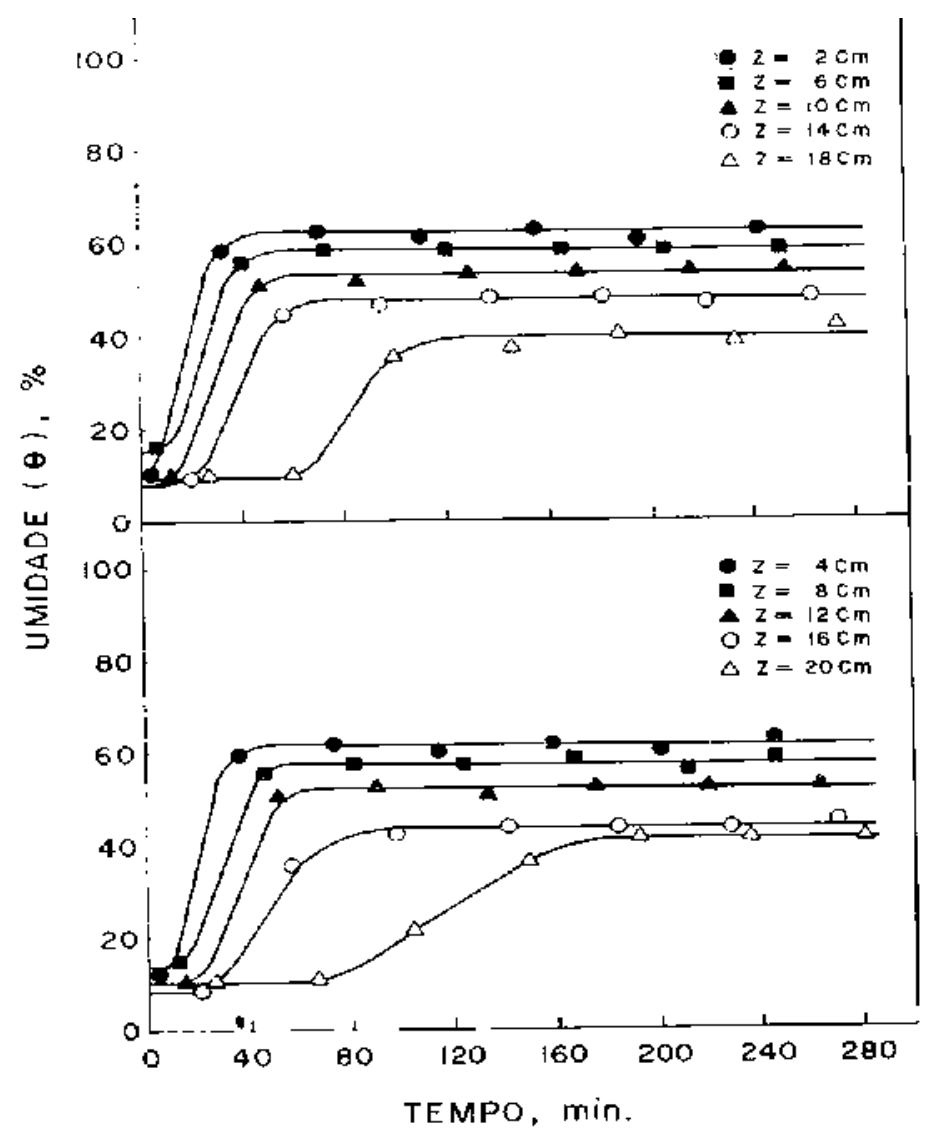

FIGURA 2 - Curvas de umidade q, obtidas para o latossolo roxo (horizonte $B)$, posições: $1(Z=2 \mathrm{~cm}) ; 3(Z=6 \mathrm{~cm})$; $5(Z=10 \mathrm{~cm}) ; 7(Z=14 \mathrm{~cm}) ; 9(Z=18 \mathrm{~cm})$ e 2 $(Z=4 \mathrm{~cm}) ; 4(Z=8 \mathrm{~cm}) ; 6(Z=12 \mathrm{~cm}) ; 8(Z=16$ $\mathrm{cm}) ; 10(Z=20 \mathrm{~cm})$.

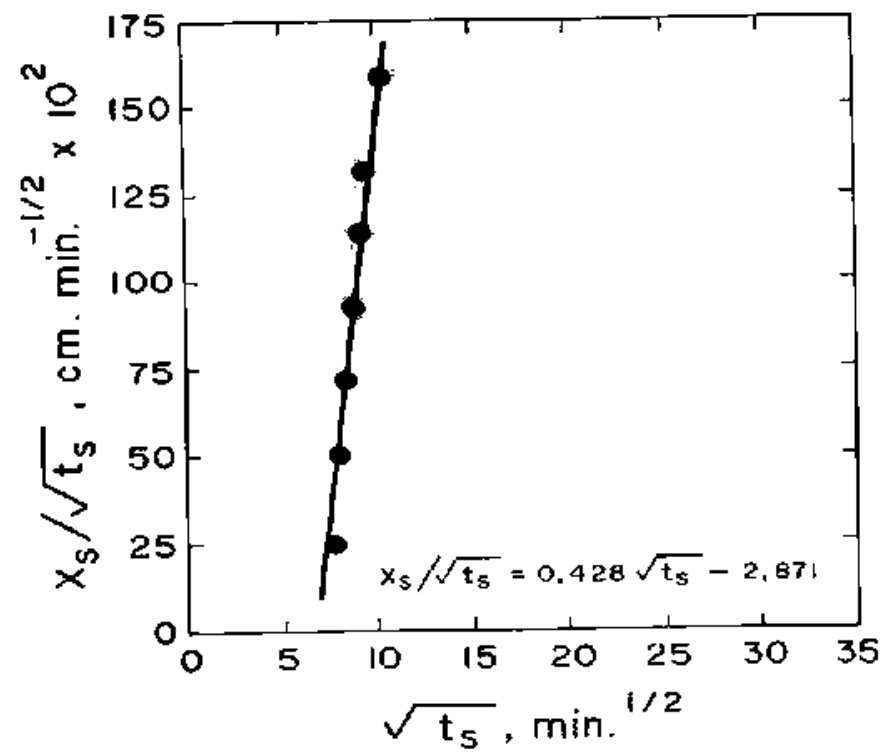

FIGURA 3 - Parametrizaçåo da evolução da frente de molhamento, apresentada pela função $x_{s} / \sqrt{t_{s}}(\mathbf{c m} x$ $\left.\min ^{-1 / 2}\right) \times \sqrt{t_{5}}\left(\min .^{-1 / 2}\right)$, obtida para o latossolo roxo (horizonte $B$ ). 


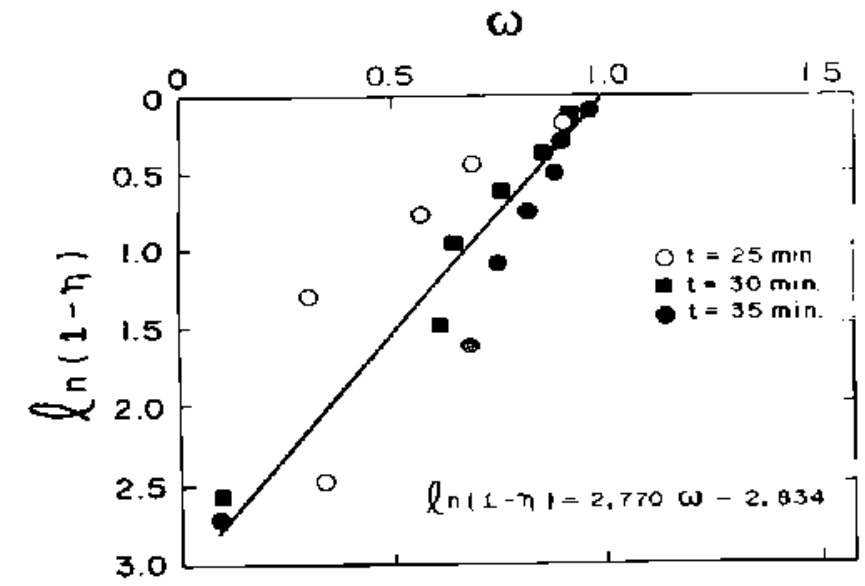

FIGURA 4 - Parametrizaçăo da funç\$o $\ell n$ para o latossolo roxo (horizonte B). Os pontos sfoo representados por simbolos. Os conjuntos de pontos de mesmo simbolo representam grupos de medidas na mesmo tempo escolhido.

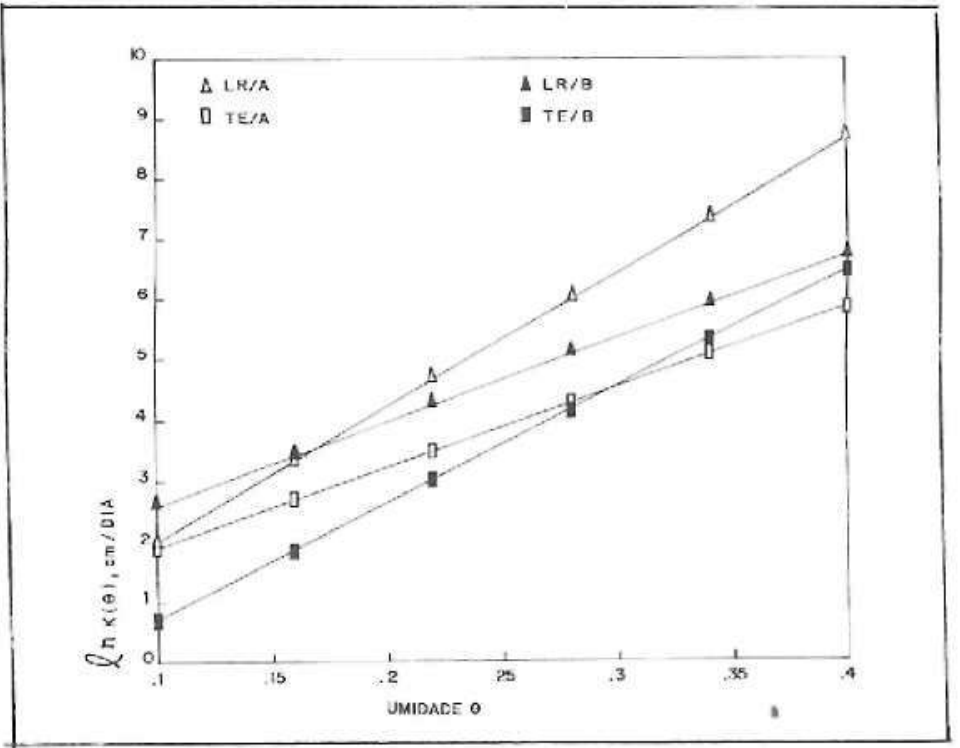

FIGURA 5 - Curvas médias da condutividade em função da umidade para os diferentes tipos de solos e horizontes estudados.

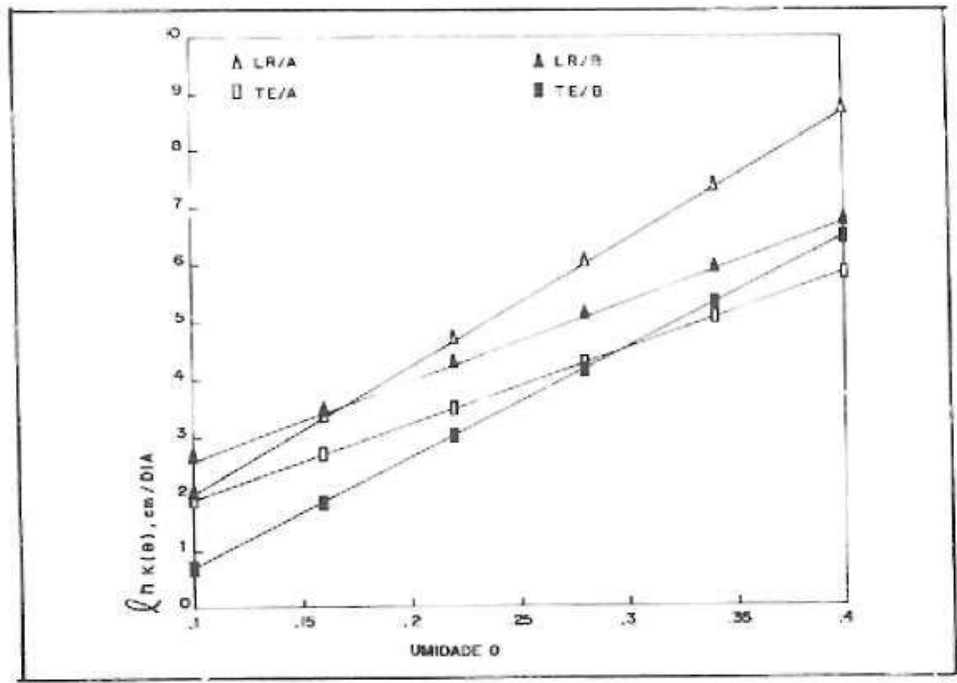

FIGURA 6 - Curvas médias da difusividade em funcão da umidade para os diferentes tipos de solos e horizontes estudados.

\section{ABSTRACT}

The hydraulic diffusivity and conductivity functions of $L R$ and $T E$ (leveis $A$ and $B$ ) soil samples from the Londrina-PR region were calculated by means of the moisture profile and data from the time evolution of the wet front, taken through measurements of the water infiltration in a soil column and a variational formulation of the vertical flow. The wet front data were taken in a acrylic column coupled in its base with a porous plate that permitted the water flow against the gravitational field with a suitable velocity of $0.12 \mathrm{~cm} / \mathrm{min}$. The moisture profile data were obtained by the gamma ray attenuation method, with a ${ }^{60} \mathrm{Co}$ source and a Nal (TL) scintillation detector. With a vertical and horizontal measurement table the moisture profile data $6(z, t)$ were taken in many points of the soil column.

KEY-WORDS: vertical infiltration, gamma ray attenuation, hydraulic diffusivity and conductivity

\section{REFERÊNCIAS BIBLIOGRÁFICAS}

1 - COUTINHO J $\mathrm{J}_{\mathrm{r}}$, G., MANIAKAS, S, DOMINGOS, R,N, \& TOBINOGA, S. Determinaçato da difusividade e da condutividade de solo nåo saturado através de dados de infilttraçf̆o vertical. Cientlfíca, Jaboticabal, v.1, p. 79-85, 1977.

2 - FERRAZ, E.S.B. Determinaçāo simultânea da densidade global e umidade do solo por afenuaçăo de raios gama do ${ }^{137} \mathrm{Cs}$ e
${ }^{241} \mathrm{Am}$. Livre Docência. ESALQ/USP. Piracicaba, SP, 1974.

3 - REICHARDT, K. Processo de transferência no sistema soloplanta-atmosfera. 4 ed. Campinas: Centro de Energia Nuclear na Agricultura/USP. Fundaçāo Cargil, 1985. Vii 466p. 